
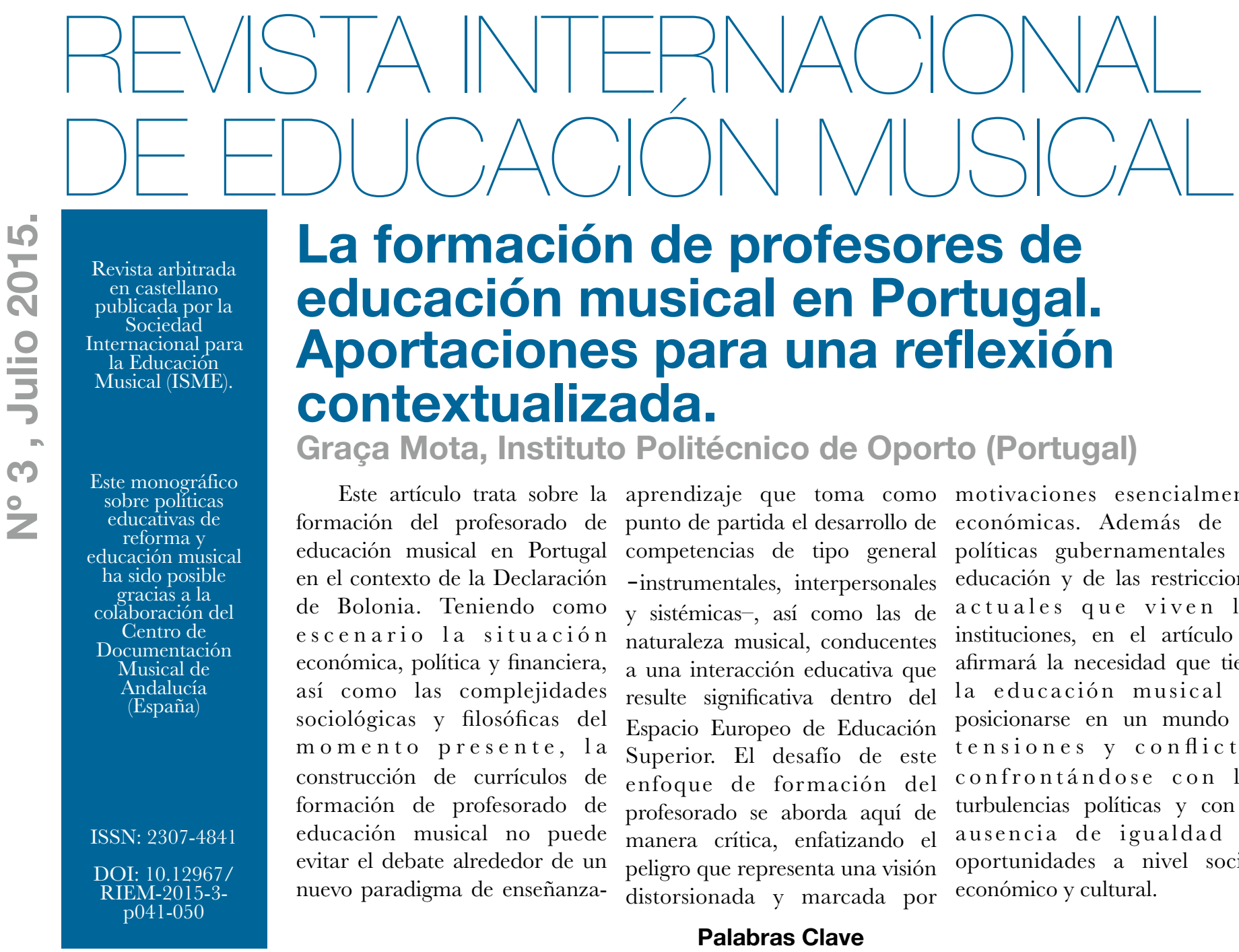

\title{
La formación de profesores de educación musical en Portugal. Aportaciones para una reflexión contextualizada.
} Graça Mota, Instituto Politécnico de Oporto (Portugal)

Este artículo trata sobre la aprendizaje que toma como motivaciones esencialmente formación del profesorado de punto de partida el desarrollo de económicas. Además de las educación musical en Portugal competencias de tipo general políticas gubernamentales en en el contexto de la Declaración -instrumentales, interpersonales educación y de las restricciones de Bolonia. Teniendo como y sistémicas-, así como las de actuales que viven las escenario la situación naturaleza musical, conducentes instituciones, en el artículo se económica, política y financiera, a una interacción educativa que afirmará la necesidad que tiene así como las complejidades resulte significativa dentro del la educación musical de sociológicas y filosóficas del momento presente, la construcción de currículos de formación de profesorado de educación musical no puede evitar el debate alrededor de un nuevo paradigma de enseñanza-

Espacio Europeo de Educación Superior. El desafío de este enfoque de formación del profesorado se aborda aquí de manera crítica, enfatizando el peligro que representa una visión distorsionada y marcada por posicionarse en un mundo de tensiones y conflictos confrontándose con las turbulencias políticas y con la ausencia de igualdad de oportunidades a nivel social, económico y cultural.

\section{Palabras Clave}

Declaración de Bolonia; formación del profesorado de música; pensamiento crítico.

\section{Music teacher education in Portugal. Contributions for a reflection in context.}

\section{Graça Mota, Porto Polytechnic Institute (Portugal)}

\section{Abstract}

This article presents the starting points the development vision, essentially moved by context of music teacher of general skills-instrumental, economical motivations. This education in Portugal after the interpersonal, and systemic-as article, beyond government Bologna Declaration. In the well as those of musical nature, educational policies and the backdrop of the present leading to a significant present institutional restrictions, economic, political and financial educational interaction within also addresses the imperative situation with its sociological and the Higher Education European that music education be philosophical complexities, the Space. The challenge of such an positioned in a world of tensions construction of curricula for approach of music teacher and conflicts, in confrontation music teacher education cannot education is addressed under a with political turbulences and avoid the discussion around a critical perspective, with an with the absence of equality of new teaching and learning emphasis on the possible dangers opportunities at the social, paradigm, which takes as that may come from a distorted economic, and political levels.

\section{Keywords}

Bologna Declaration; music teacher education; critical thinking. 


\title{
La formación de profesores de educación musical en Portugal. Aportaciones para una reflexión contextualizada.
}

\author{
por Graça Mota, Instituto Politécnico de Oporto (Portugal).
}

En este capítulo reflexiono sobre la formación del profesorado de educación musical en Portugal enmarcándola en la declaración de Bolonia y en la creación de un Espacio Europeo de Enseñanza Superior (EEES). Voy a intentar compartir con los lectores una perspectiva de educación musical para toda la ciudadanía partiendo de un concepto democrático de acercamiento al saber musical que se asienta en un abordaje crítico que tiene en cuenta el mundo de hoy y, más concretamente, el contexto europeo.

En una primera parte haré una introducción histórica a propósito de la educación musical en el ámbito del sistema educativo portugués y del establecimiento de las bases para la formación de profesores de Enseñanza Básica (EB) en este campo del saber desde 1986 hasta la llegada, en 2006, del plan Bolonia y su aplicación en la Educación Superior (ES). Seguidamente procuraré identificar los desafíos actuales para una educación musical democrática, así como para encontrar perspectivas para la formación del profesorado de educación musical basadas en el concepto de sostenibilidad. La última parte del artículo estará dedicada a una reflexión sobre el marco legal actual creado a partir de la declaración de Bolonia y sobre el abanico de oportunidades que dicha Declaración podría crear para una reconstrucción curricular de la educación superior. Estas cuestiones estarán influenciadas, lógicamente, por la coyuntura social, económica y política que Europa está atravesando y por el alcance que esta política ha tenido a nivel de la educación superior con especial incidencia en la formación del profesorado en artes.

\section{La Educación Musical en el sistema educativo portugués}

La educación en general y la musical en particular han sufrido modificaciones radicales a partir del establecimiento de la democracia en Portugal en 1974. El fin de la dictadura trajo consigo una amplia discusión sobre cuál debería ser el papel de la educación formal en la formación de un ciudadano pleno en una sociedad conformada por los valores democráticos. En esos tiempos conturbados y al mismo tiempo ricos de la vida portuguesa, la música tuvo un papel significativo en el plano social y político pero no constituía una prioridad desde el punto de vista educativo. Muchos jóvenes se identificaron entonces con la llamada música de intervenção cuyos textos reflejaban claramente las preocupaciones de la sociedad portuguesa y tuvieron un papel relevante en el proceso revolucionario que estaba en curso (Mota, 2001). Mucha de esta música también llegó a tener una expresión significativa dentro de las escuelas y diferentes grupos de música pop portuguesa la siguen tocando todavía, si bien con nuevos arreglos y, evidentemente, menos motivada por la llama revolucionaria. Es interesante verificar que en las condiciones socioeconómicas actuales una parte de esta música ha llegado a ser recuperada de acuerdo con las cuestiones más

candentes que representan las grandes preocupaciones de la sociedad portuguesa.

A la hora de abordar la educación musical en el Portugal de hoy consideraré, aunque brevemente, la distinción entre la educación musical general para toda la ciudadanía y la educación especializada de la música, con carácter vocacional. Para ello, me ayudaré de la legislación que se fue produciendo y que estableció el marco legal para estas dos vertientes de la enseñanza de la música.

Hasta la publicación en 1983 de la ley 310/83 (Portugal, 1983), todos los profesionales de la música se formaban en los conservatorios y en las academias de música obteniendo un diploma final en instrumento, composición o canto, sin contemplar ninguna especie de estudios educativos. A partir de la reglamentación de 1983, este sistema vertical de enseñanza que permitía que en una misma institución se procesara la educación básica, la secundaria y la superior de música dejó de tener lugar. La enseñanza básica y secundaria se mantuvo en los conservatorios y en las academias mientras que la enseñanza superior (ES) pasó a estar bajo la tutela de los Institutos Politécnicos (Escuelas Superiores de Música y Escuelas Superiores de Educación) y de las Universidades. Las Escuelas Superiores de Música fueron las encargadas de graduar a los músicos profesionales y a los profesores de música para los conservatorios y academias. A las Escuelas Superiores de Educación se les encomendó la graduación de todos los profesores para la EB, incluidos los profesores de Educación Musical.

El aspecto innovador de la reglamentación de 1983 residió en el acto de reconocer que la formación de un músico, tanto para la enseñanza general como para la enseñanza vocacional, tendría que integrar no solo un currículo de música altamente especializado a nivel del instrumento, del canto o de la composición, sino también a nivel de las ciencias de la educación y de las cuestiones culturales, sociológicas, filosóficas y estéticas específicas de la música (Mota, 2003ª).

\section{La Educación Musical en la EB}

La Educación Musical en la EB sufrió un cambio radical como resultado de la reforma nacional del sistema educativo y la publicación de la Ley de Bases del Sistema Educativo (LBSE) en 1986 (Portugal, 1986) que introdujo los títulos de graduación en Educación Musical en las Escuelas Superiores de Educación de los Institutos Politécnicos.

En las últimas décadas del siglo XX y del inicio del siglo XXI, los debates en el seno de la comunidad de educadores musicales en torno a los nuevos currículos de música para la EB contribuyeron a agrandar las perspectivas acerca de lo que debería ser lo esencial de la educación musical y, consecuentemente, de la formación del profesorado para este área del saber. En este ámbito, el pensamiento de educadores musicales contemporáneos tales como Benett Reimer, Keith Swanwick y, más recientemente, David Elliott, Lucy Green o Estelle Jorgensen, entre otros, formó parte integrante de este 
debate y contribuyó a iluminar los diferentes aspectos de lo que se entiende hoy por una educación musical de cariz moderno y abierto a las diferentes corrientes del pensamiento sociológico, filosófico y político.

En este sentido, la reforma curricular que siguió a la publicación de la LBSE puso particular atención en la formación del profesorado de educación musical, disciplina que pasó a formar parte del Currículo Nacional, obligatoria para todos los niños y jóvenes de 7 a 14 años de edad. Sin embargo, los sucesivos Ministerios de Educación se remitieron en la práctica a una posición ambigua en la que las decisiones importantes fueron aplazadas sistemáticamente, impidiendo de forma irreversible que la educación musical se estableciese de pleno derecho en paridad con las otras materias curriculares.

En el primer ciclo de la EB, sobre todo en los primeros cuatro años de escolarización, la música llegó a estar implementada de modo sistemático. Aunque la LBSE en su artículo $8^{\circ}$ preconice para este ciclo de la EB la presencia de profesorado auxiliar en las áreas artísticas, como por ejemplo para la Expresión y la Educación Musical, esto no llegó nunca a ser puesto verdaderamente en práctica. Los profesores de enseñanza general, responsables de la enseñanza de todas las disciplinas, también han de enseñar en las áreas artísticas, incluyendo la educación musical. Sin embargo, esta expectativa nunca se cumplió adecuadamente en la medida en la que este profesorado posee una formación musical muy limitada, formación que tiene como consecuencia una falta de confianza generalizada que les merma a la hora de llevar a cabo un trabajo consecuente y secuencial a lo largo de los cuatro años de aprendizaje bajo su responsabilidad. De este modo, el desarrollo musical de los niños y niñas en este nivel de escolarización fue dejado más o menos al azar o al interés particular de los padres, al de algunos profesores empeñados y al de la comunidad.

En 2006, el Ministerio de Educación publicó una ley que establece la educación musical como una actividad extracurricular, o sea, se asumió tácitamente que, como materia curricular no había sido implantada como sería deseable (Mota, 2007; Boal-Palheiros y Encarnação, 2008). En realidad, la situación en las escuelas demuestra que el profesorado de enseñanza general sigue teniendo una baja autoestima respecto a sus capacidades para llevar a cabo la educación artística en general y la educación musical en particular, además de la clara percepción de que sus competencias musicales están lejos de las exigencias curriculares (Mota, 2003 ${ }^{\mathrm{a}}, 2003^{\mathrm{b}}, 2^{2003^{c}}$ ).

En el segundo ciclo de la EB, que corresponden al quinto y sexto año de escolaridad, la educación musical tiene su lugar asegurado en el currículo: una hora y media a la semana a cargo de un profesor especializado. Este es el momento en el que esta disciplina se trabaja de forma secuencial, aunque no siempre el desarrollo musical de los niños esté de acuerdo con sus edades. Esto parece ser una clara consecuencia de la falta de enseñanza musical sistemática en los años anteriores, y habrá que investigar los resultados de la implantación del área extracurricular de música en el primer ciclo, previamente citado, para la cual no existen todavía datos relevantes. Es necesario, no obstante, reconocer que un número significativo de niños está motivado por las experiencias musicales que tienen lugar en estos dos años de escolarización, y encara la música como una posible opción para una profundización ulterior en dichos estudios. Igualmente es en este nivel de enseñanza en el que el profesorado de educación musical forma grupos regulares de práctica instrumental y vocal, lo que determina una práctica musical regular en las escuelas (como, por ejemplo, actuaciones en público y clubs musicales).

En el tercer ciclo de enseñanza básica -séptimo, octavo y noveno año de escolaridad-, la educación musical tiene una expresión desigual en todo el país. Las escuelas que ofrecen esta disciplina tienen la oportunidad de desarrollar los conocimientos musicales previamente adquiridos por los jóvenes a través de actividades de interpretación instrumental y vocal, composición y arreglo y, de acuerdo con las disponibilidades, de tecnología musical. Desgraciadamente, los más recientes desarrollos en la reforma curricular se están traduciendo en cortes sistemáticos, sobre todo en las artes, lo que llevó a que en el último año del tercer ciclo, noveno curso, la música dejara de estar presente en el currículo (Portugal, 2011).

A modo de resumen de esta sección, me gustaría mencionar que, a pesar de los diferentes factores que dificultan el acceso de los niños, niñas y jóvenes portugueses a la educación musical en el contexto formal, un número significativo de actividades musicales tienen lugar fuera del sistema educativo formal, muchas veces hasta como una prolongación de dicho contexto (Mota, 2008a, $2008^{b}$ ). Por otro lado, los educadores musicales toman cada vez más conciencia de esta realidad e intentan salvar el foso existente entre las prácticas musicales de dentro de la escuela y las de fuera, implicándose y promoviendo la implicación en comunidades de práctica que representan un papel muy importante en el día a día de los jóvenes (Wenger, 2006).

\section{La enseñanza especializada de la música}

En el sistema educativo portugués coexisten tres tipos de escuelas de enseñanza especializada de la música: los conservatorios (financiados por el Estado), las academias de música y las escuelas profesionales de música (sostenidas por el sector privado con apoyo del Estado).

Como ya hemos mencionado, los conservatorios y academias de música fueron objeto de reglamentación en 1983, perdiendo su carácter de enseñanza vertical en tanto que únicas instituciones de formación de profesionales de música. Hoy en día representan un complemento a la enseñanza general y ofrecen los ocho cursos del currículo secundario nacional para instrumento, canto, teoría musical y composición, además de otras enseñanzas libres para aquellos estudiantes que no pretenden seguir estudios musicales de nivel superior.

Las escuelas profesionales de música, por su parte, habían surgido en 1989 con el objetivo de ampliar la oferta de formación musical a dos niveles: a) mediante la incentivación por parte de las entidades privadas presentes en el sistema; b) mediante la promoción de una autonomía curricular y pedagógica frente al Estado. Estos objetivos reflejaron claramente el zeitgeist político de la época al aparecer en el contexto de un "cambio y modernización del tejido social que [existía] no solo en Portugal sino también en la comunidad europea y en los países de la OCDE" (Portugal, 1996, p. 8). La idea fundamental de estas escuelas era la de establecer una clara distinción con respecto a las otras instituciones de enseñanza especializada de la música en un intento por preparar instrumentistas capaces de 
llegar a formar parte de las orquestas portuguesas. Aunque sobrevivieran, en sus inicios fundamentalmente a través de la contratación de un gran número de músicos extranjeros, el hecho es que hoy en día tienden a contar, cada vez más, con músicos portugueses ${ }^{1}$.

$\mathrm{Al}$ final de esta sección es necesario referir, una vez más, la interesante posibilidad de articulación entre las escuelas de enseñanza especializada de la música (conservatorios y academias de música) y la EB, que fuera reglamentada a través de una legislación específica (Portugal, 2002). En ella se determina, básicamente, que el alumnado que ingresa en el segundo ciclo de enseñanza general (quinto y sexto años de escolarización) y que asisten a la vez a un conservatorio o una academia de música pueden disfrutar de las ventajas de un programa articulado que incluye partes fundamentales del currículo general y del currículo específico de la música en un proceso que puede darse en una u otra institución. A un nivel meramente práctico esto significa una gestión más racional del calendario escolar del niño. A un nivel más elevado, tiene la capacidad de permitir la colaboración institucional en proyectos artísticos comunes. En realidad, y aunque todavía sean muy pocos los estudios llevados a cabo en este campo, parece que se dan colaboraciones de ese tipo en los pocos casos en los que directores y profesores reconocen las ventajas de una integración con ambos sistemas educativos (Madeira, 2012).

\section{La formación del profesorado de educación musical}

Después de la legislación de 1983 y 1986, y como ya he mencionado, las Escuelas Superiores de Educación iniciaron una oferta de formación del profesorado de educación musical para la EB. Se inició un proceso irreversible por primera vez en Portugal que se concretaba en una graduación tras cuatro años cuyo currículo correspondía a un perfil de profesor de enseñanza general para el primer ciclo de la EB y de profesor especializado en música para el segundo. Este hecho aportó una renovación significativa a nivel de la música para toda la ciudadanía en el ámbito de la escuela pública. Aunque todavía no haya estudios sistemáticos sobre la relación entre la enseñanza general de la música y la consiguiente frecuencia de la enseñanza especializada, el hecho de que un número tan elevado de niños y niñas acudan hoy en día a los conservatorios y academias de música puede ser consecuencia de una educación musical de mayor calidad en la enseñanza general (Fernandes, 2007).

Esta formación del profesorado estuvo en vigor hasta la llegada del plan Bolonia, cuyo marco legal (Portugal, 2006; Portugal, 2007) supuso cambios importantes en lo que respecta a la definición de habilitación profesional para la docencia, obligando a que la misma sea conferida, únicamente, a quien posea una licenciatura en ese campo específico seguida de un máster específico. En la Escuela Superior de Educación del Instituto Politécnico de Oporto, por ejemplo, fueron creadas de este modo, entre otras, la Licenciatura en Educación Musical y el Máster en Enseñanza de la Educación Musical.

\section{En busca de una Educación Musical democrática. Los retos del plan Bolonia.}

Me gustaría enfatizar especialmente el pluralismo y la heterogeneidad, todo aquello a lo que hoy se llama multiculturalidad. Y mi elección tiene como pretensión relacionar las artes con una comunidad siempre en acción, una comunidad a la que un día podremos llamar democracia (Greene, 1995, p. 6).

Todo proceso de reestructuración curricular que tenga lugar en una institución solo podrá ser llevado a cabo en el contexto de un debate más amplio que tenga en cuenta la experiencia acumulada de la implantación de un currículo dado, conjugándolo con la definición de los valores educativos subyacentes a la reestructuración ahora pretendida. Así, y siguiendo la perspectiva de Maxine Greene, se hace necesario resistir cuando se lucha por la construcción de una narrativa que examina y reexamina los procesos humanos a propósito de cuestionamientos que, si bien abordan la educación, la educación musical o cuestiones de orden curricular, se hace como si estuviesen desligadas de la vida, de la experiencia y de la lucha por la igualdad de oportunidades y de la justicia en lo público. En la misma línea de pensamiento, la redefinición de lo que se entiende por educación musical en la ES en Europa tendría que darse junto con el reconocimiento de que la educación no puede ser un acto neutral frente a los enormes desafios que hoy se nos ponen y que determinan nuestro malestar ante contextos de injusticia, desigualdad y violencia.

Consecuentemente, la búsqueda de una educación musical democrática necesita de una reflexión plural para la que todos somos indispensables. Me parece importante seguir de cerca los desarrollos recientes en el sentido de establecer una conexión entre la música y los movimientos que abordan los conflictos mundiales y luchan por la paz. Bergh y Sloboda (2010) nos dan una excelente revisión de la literatura en cuanto a la utilización de la música como agente de transformación y promoción de la paz en el ámbito de varios conflictos mundiales. También el legado de Dewey (1916/2007) nos ayuda a comprender el sentido de una «educación [musical] democrática» al establecer la conexión entre educación, democracia y reforma social. En palabras de Giroux (1989, p. 184), Dewey...

[...] nos recuerda que la educación puede funcionar, bien sea en el sentido de crear ciudadanos pasivos e incapaces de correr riesgos, o bien en el sentido de generar una ciudadanía políticamente educada capaz de luchar por las diferentes formas de una vida pública preocupada por la justicia, la felicidad y la igualdad.

Estas ideas encuentran también eco en las preocupaciones de Bowman (2007) cuando afirma la necesidad de situar las cuestiones de la justicia social dentro de la música y, de este modo, dentro de la educación musical:

La música ya es siempre cultural y social (y también política, a mi entender). Así, abordar $-\mathrm{O}$ no- problemas de equidad y de justicia social no es la verdadera cuestión. Las cuestiones reales son: a) cuáles son los intereses a los que estamos sirviendo cuando excluimos o ignoramos esos problemas; b) qué tipo de músicas, valores, percepciones y personas han sido excluidos mediante nuestras tácticas territoriales (p.119).

Partiendo de estas premisas, ¿qué puede significar hoy en día una reconstrucción curricular de la educación musical en la ES, teniendo como telón de fondo el plan Bolonia y sus objetivos, seguidamente enunciados?

- Construcción de un área competitiva en el EEES.

- Promoción de la movilidad y de la empleabilidad en el EEES.

- Sistema similar de créditos (ECTS). 
- Tres ciclos de estudios.

- Un sistema similar de grados académicos.

- Movilidad de estudiantes y profesores.

- Cooperación europea para la certificación y garantía de calidad.

- Promoción de una dimensión europea curricular y de investigación.

La ley portuguesa que reguló la implantación del plan Bolonia (Portugal, 2006) reconoce que la cuestión central es un cambio de paradigma. Se trata de abandonar un modelo pasivo de adquisición del conocimiento con vistas a otro basado en el desarrollo de competencias, bien sea de sentido genérico -operacionales, interpersonales y sistémicas- o bien asociadas al campo específico de aprendizaje. Un aspecto fundamental a tener en cuenta es el trabajo basado en proyectos para la promoción progresiva de la autonomía del estudiante.

La implantación del plan Bolonia ha sido hecha en todo el territorio europeo de manera muy desigual y, particularmente en Portugal, con enormes variaciones de una institución a otra. Las relaciones entre el Ministerio de Educación y las Universidades e Institutos Politécnicos revelan una ausencia de liderazgo político, lo que tiene como consecuencia, a veces, una visión distorsionada y esencialmente marcada por unas intencionalidades ocultas (económica y financiera). Así, una buena parte de las instituciones se ve impelida a dar prioridad a objetivos de orden táctico en detrimento de los aspectos científicos y pedagógicos. Este es el lado oscuro y mercantil del plan Bolonia.

El deseado cambio de paradigma y la promoción de una significativa revolución pedagógica necesitan de una evaluación crítica del siguiente conjunto de parámetros, deseables para una auténtica reconstrucción curricular:

- Flexibilidad curricular. Los estudiantes deberían tener más libertad para construir sus propios currículos dentro de un sistema modular que permitiera diferentes opciones dependiendo de los intereses específicos o bien una especialización en el ámbito del primer ciclo de estudios (licenciatura) con vistas a un posible postgrado en un segundo ciclo de estudios (máster). Este es un aspecto que ha tenido poca o ninguna concreción, ya que la mayoría de las instituciones sigue ofreciendo carreras sin espacio para que los estudiantes ejerzan cualquier autonomía curricular.

- Otra organización del trabajo entre el profesor y el estudiante. Tanto el profesorado como el alumnado tendrían que adaptarse al nuevo paradigma. Esto significa que el profesorado tendría que preparar más material para tener a disposición de sus estudiantes y que estos habrían de desarrollar un mayor sentido de la autonomía en su trabajo, lejos del ambiente de la enseñanza y del aprendizaje presencial. En términos de educación musical, este es el cambio más significativo tanto en términos filosóficos como prácticos, ya que tienen en cuenta el ansia de los estudiantes por explorar nuevos y desafiantes mundos musicales (Jorgensen, 2003, 2008, 2011; Green, 2006).

- Trabajo tutorial. La tradición del trabajo tutorial en la ES no es una realidad en algunos países europeos. Por ejemplo, contrariamente al Reino Unido, Portugal tiene una larga tradición de clases magistrales presenciales en detrimento del trabajo independiente de los estudiantes con acompañamiento tutorial. Cambiar este status quo representa, en realidad, un gran desafío. Poner a los estudiantes en el centro de la organización de su propio aprendizaje es un cambio de paradigma que implica: 1) una diferente propuesta curricular para la formación del profesorado; 2) una mayor flexibilidad de organización de los horarios de los profesores y del curso académico; 3) estructuras institucionales de apoyo que apoyen tanto a los profesores como a los estudiantes en el desarrollo de estas nuevas condiciones de trabajo.

- Menor ratio profesor-alumno. En las últimas décadas, las instituciones de ES han estado bajo permanente presión para que se aumente el número de estudiantes por aula. Igualmente sucede en las artes y en la música, donde se reconoce que el número de alumnos debería ser menor, pero la necesidad de reducir costos empieza a hacerse sentir. La lógica de Bolonia con su llamada a un nuevo paradigma de enseñanza no es compatible con unos propósitos financieros. Sin embargo, e incluso en momentos particularmente difíciles desde el punto de vista económico, es absolutamente necesario buscar soluciones alternativas de acuerdo con la especificidad de cada materia. La utilización de interacciones diversificadas y creativas entre profesorado y alumnado puede incluir la comunicación vía web o el uso de la tecnología musical de la que hay ejemplos interesantes en el Reino Unido, en la Guildhall School of Music and Drama y en el Royal Northern College of Music $^{2}$, por ejemplo.

- Articulación entre aprendizaje e investigación. Uno de los cambios más significativos que deberían tener lugar en las instituciones de ES sería la comprensión de que el proceso de aprendizaje tiene que estar profundamente ligado a la investigación. Este aspecto es particularmente relevante en los programas de formación del profesorado. Si creemos que la investigación es un instrumento poderoso para comprender el mundo, generar nuevas ideas y cambiar la vida, la ES tiene una mayor responsabilidad a la hora de establecer la comunicación entre el conocimiento y la práctica.

En definitiva, la necesidad anteriormente sugerida de una revolución pedagógica puede ahora resumirse en la reconstrucción de la educación en tres niveles: cultural, éticopolítico y cívico. A propósito de esto, Nóvoa (2007) propone temas obligatorios para una reflexión e intervención en la educación: por un lado, la consideración de la diversidad como una forma de redefinición de prácticas de inclusión social y de integración -asumiendo la imposibilidad actual de hablar de un único modelo educativo- y, por otro lado, los desafíos que afrontamos hoy en día frente a las llamadas nuevas tecnologías y su inmensa importancia en la formación del profesorado.

\section{En busca de un concepto de sostenibilidad en la formación del profesorado de educación musical}

En este apartado voy a referirme a la construcción de un concepto de sostenibilidad para la formación del profesorado de educación musical en el contexto del EEES considerando los tres principios recíprocos de la actividad educativa tal como fueron elaborados por Abbs (2003). 
El primer principio -la educación es existencial en su naturaleza- apunta al hecho de que la educación no puede tener lugar sin la participación activa de los estudiantes. Abbs considera que existir es "hacernos visibles, declararnos, confesarnos, ejercer nuestra libertad a la vez que ser agentes de nuestras propias acciones y comprensión” (ibíd., p. 15). Las consecuencias de este principio en los programas de formación del profesorado de educación musical son el reconocimiento de que la biografía musical de nuestros estudiantes no puede excluirse de todos los momentos musicales que tienen lugar en el espacio educativo. Esta idea nos remite nuevamente a la contribución de Greene (1995) sobre el descubrimiento de una pedagogía que promueva la posibilidad de dar a los jóvenes el poder de abordar y construir múltiples realidades, comenzando por la capacidad de enunciación de sus propios mundos.

El segundo principio - la educación es esencialmente una actividad colaborativa - sustenta la idea de que no existimos sin el otro y, en consecuencia, de que el individuo que se encuentra en un proceso educativo necesita estar integrado en una comunidad. Abbs considera que el acto educativo no puede tener lugar en ausencia de diálogo, sin explorar las condiciones de los otros en el sentido de poder alcanzar una comprensión común. La conclusión a la que se llega en cuanto a este principio es que las actividades musicales tienen que ser concebidas como momentos de colaboración e interpretación a nivel de la apreciación musical, de la actuación, de la improvisación y de la composición.

El tercer principio -la educación es siempre una actividad cultural que se debe expandir y en la que se debe profundizar continuamente-, Abbs lo entiende como una ampliación de los principios existenciales y colaborativos anteriores y, por eso, propone una iniciación progresiva de los estudiantes en la cultura de una disciplina dada, en este caso la música:

De acuerdo con este tercer principio, la educación existe para poner en marcha una conversación entre varias generaciones y a través de las diferentes culturas, de los tiempos y de los espacios a fin de que los estudiantes se vean desafiados por otras formas de comprensión y, al mismo tiempo, tengan acceso a todo tipo de materiales -metáforas, modelos, ideas, imágenes, narrativas, actosDe este modo tendrán la posibilidad de configurar, reconfigurar y volver a probar ese proceso interminable que son sus propias vidas intelectuales y espirituales (ibíd., p. 17).

Para un programa de educación musical, este último principio evoca la necesidad de sobrepasar barreras, de moverse a través de diferentes épocas, estilos y géneros musicales en un proceso en el que el profesor tendrá que ayudar a sus estudiantes a establecer las conexiones necesarias en el sentido de construir un tejido cultural. Junto con los otros dos principios, y desde mi punto de vista, hay que crear un marco referencial en el que la sostenibilidad sea construida y reconstruida en un proceso de apropiación por parte del alumnado y del profesorado, garantizando así la posibilidad de un desarrollo de aprendizaje autónomo y continuado a lo largo de la vida.

\section{Una propuesta curricular}

En el ámbito de la reestructuración curricular que tuvo lugar en las instituciones portuguesas de ES como consecuencia de la reglamentación del plan Bolonia, mi institución, la Escuela Superior de Educación del Instituto Politécnico de Oporto inició un proceso de revisión curricular que, tal y como ya he referido, dio lugar a la creación de la Licenciatura en Educación Musical y del Máster en Enseñanza de la Educación Musical.

Las discusiones previas a este trabajo buscaron capitalizar nuestras experiencias tanto positivas como negativas de los 20 años de experiencia de la licenciatura anterior teniendo en cuenta un conjunto de parámetros que seguidamente se sintetizan en tres puntos:

1. Un currículo centrado en la actividad musical. En esta idea subyace una comprensión de la organización de toda la formación inicial del educador musical en torno al acto de hacer música en sus múltiples aspectos, ya sea orientado a la práctica musical individual o colectiva, o como una forma de enmarcar las actividades musicales a desarrollar con niños. Esta comprensión incluyó un entendimiento ampliado de las cuestiones clave que se tratan hoy en día en las ciencias de la educación sobre su aplicación concreta al acto de enseñar y aprender. Dicha comprensión solo podrá promoverse en el contexto de actividades de iniciación a la práctica pedagógica en ambientes formales, informales y no formales. Por otro lado, el desarrollo de competencias musicales debería estar estructurado en el sentido de que es una actividad musical de múltiples facetas y múltiples objetivos -actuación, improvisación, composición y arreglo y producción-. Estas facetas y objetivos deberían seguir formando parte de las cuestiones esenciales de las ciencias musicales tales como la historia de la música, el análisis cultural, la sicología de la música, la estética musical y la tecnología musical. Finalmente, nos parece indispensable que los futuros educadores musicales desarrollen las competencias necesarias para una enseñanza reflexiva a través de una introducción a la práctica informada por la investigación.

2. La integración de la acción y su significado. Basándonos en el punto 1 intentamos estructurar el currículo en una unión sistemática entre teoría y práctica, tanto en espacios de aprendizaje musical teórico y práctico como en proyectos en los que el conocimiento pueda ser experimentado, interpretado y contextualizado. Tal y como dice Dewey (1934), la obra musical, en tanto que obra de arte, adquiere aquí otra dimensión como experiencia vivida transformada por los sujetos que la produjeron. Esta idea fue decisiva en nuestro plan de estudios para la propuesta de creación de un espacio de artes integradas, un laboratorio de experimentación de educación artística a través de la exploración de diferentes medios desde una perspectiva educativa en tanto que "proceso para capacitar a las personas para la diferencia y para que se introduzcan en las múltiples regiones de significado que crean perspectivas en las obras de arte" (Greene, 2001, p. 5).

3. La autonomía. El nuevo currículo, basándose en las propuestas de Bolonia para la movilidad estudiantil y en colaboración interinstitucional, tendría que permitir que cada estudiante, futuro educador musical, encontrase su personalidad musical y desarrollase su imaginación con el fin de saber ver y comprender nuevas situaciones y ser capaz de proponer alternativas.

Considerando estos tres ejes curriculares, nuestros grados y postgrados tienen como objetivo la educación de 
profesionales de la música con las siguientes competencias que los deben capacitar para:

- Integrar contextos educativos informales, no formales y formales apoyando sus intervenciones en las teorías y prácticas en educación musical actuales.

- Construir proyectos artísticos que, estando fuertemente anclados en la música, puedan y deban integrar manifestaciones de otras artes.

- Contextualizar su intervención dentro de una perspectiva de trabajo colaborativo entre todos los elementos implicados en el ambiente educativo y en el respeto por la diversidad cultural y personal.

- Tener en cuenta las prácticas socioculturales y artísticas de una comunidad concienciada, reconociendo su relevancia formativa y educativa.

Estos cambios deseables en el desarrollo curricular para los grados y los posgrados apuntan claramente a un nuevo paradigma que está siendo evaluado regularmente por parte de entidades internas y externas. Reconocemos, sin embargo, que esto deberá ir a la par con una nueva forma de mirar al profesorado y su papel en la sociedad.

\section{Resituar el papel del profesorado}

La importancia de una política dirigida al profesorado está bien patente en el informe de la OCDE, Teachers Matter, publicado en 2005:

Los profesores son importantes por el impacto que tienen en el aprendizaje de sus alumnos. Las investigaciones indican que aumentar la calidad de los profesores es tal vez la directiva política más capaz de llevar una mejora sustancial en las prácticas escolares. Por eso hay aspectos de la calidad de un profesor muy importantes que no se traducen en indicadores -tales como sus cualificaciones, su experiencia y exámenes de capacitación académica-. Las características del profesorado que son más difíciles de medir y que, sin embargo, son vitales para el aprendizaje de los alumnos, necesitan ocupar más espacio en la formación y empleo de los docentes (OECD, 2005, p. 23).

Para hacer frente a los desafios de una educación musical tal como viene siendo defendida en las anteriores secciones de este artículo, los profesores y profesoras son absolutamente insustituibles no solo para promover un ambiente de aprendizaje significativo, sino también para desarrollar procesos de integración en una sociedad cada vez más diversificada culturalmente. Claro que, como reconoce Nóvoa (2007), al tiempo que Europa precisa cuidar del desarrollo profesional de los docentes, está profundamente preocupada con el hecho de una producción excesiva de discursos sin que estos tengan una traducción en la práctica: "tenemos un discurso coherente -en muchos aspectos consensuado-, estamos de acuerdo en cuanto a lo que hay que hacer pero escasamente hemos conseguido hacer aquello que decimos que es preciso hacer" (p. 4).

Este autor (ibíd.) propone un programa de acción que incluye tres ideas: 1) aproximar y vincular a la «comunidad de formadores de profesores» y a la «comunidad de profesores»; 2) promover nuevas formas de organización dentro de la profesión; 3) reforzar la presencia personal y pública del profesorado en la sociedad. Voy a abordar brevemente estas tres orientaciones en relación con la comunidad de profesores y profesoras de educación musical y en una deseada relación con las ideas que han ido desarrollándose en las anteriores secciones.
En relación con la primera idea, Nóvoa refiere el largo divorcio entre las universidades $-\mathrm{y}$ el mundo académico en general- y la comunidad de docentes que trabajan en sus escuelas. Mientras la producción teórica sobre la formación del profesorado es amplia y significativa, poco se sabe sobre los problemas y dificultades a los que los profesores tienen que hacer frente en su día a día escolar (OECD, 2005). Si verdaderamente queremos encarar los desafíos -tal como hemos presentado en este artículo- y las ideas de académicos como Maxine Greene, Wayne Bowman y Peter Abbs, se hace urgente contribuir a una mayor intersección entre las dos comunidades. Y no hay que hacerlo de forma meramente teórica, sino creando oportunidades tanto en la formación inicial como en la continua de los docentes. Tal como sugiere Nóvoa (2007, p. 7):

[Estas propuestas] solo tienen sentido si han sido construidas dentro de la profesión, si han sido apropiadas a partir de una reflexión de los profesores sobre su propio trabajo. Mientras solo sean imposiciones desde el exterior, los cambios que tengan lugar en el interior del campo profesional docente serán bien pobres.

La segunda idea -la promoción de nuevas formas de organización dentro de la profesión- está desarrollada en torno a la imposibilidad de reforzar la profesión si no existen fuertes comunidades de práctica en las que el profesorado pueda interactuar y compartir ideas. Nóvoa expone la fragilidad actual de los movimientos pedagógicos que tuvieron durante décadas un papel central en la innovación de la educación y cita a Hutchings y Huber (2005) a este respecto. En realidad, estos autores defienden la necesidad de compartir el trabajo académico en el campo de la enseñanza y del aprendizaje diciendo en «comunidades de enseñanza»:

Un espacio conceptual en el que las comunidades de educadores dedicados a la investigación e innovación se juntan para intercambiar ideas sobre la enseñanza y el aprendizaje, utilizándolas para ir al encuentro del reto de educar a los estudiantes para la vida personal, profesional y ciudadana (p. 1).

En Portugal, este tipo de comunidades de práctica comienza a emerger sobre todo entre jóvenes educadores musicales y en su mayor parte relacionadas con prácticas de tecnología musical en la clase de educación musical. Pienso que es muy importante que la ES se mantenga en contacto con esta y otras prácticas semejantes, promoviendo la discusión sobre metodologías y métodos que puedan mejorar e informar el acto de hacer música en el ámbito de la Educación Musical en la EB.

Finalmente, considerando la tercera idea de Nóvoa -reforzar la presencia personal y pública de los profesores en la sociedad-, me gustaría regresar al informe de la OCDE previamente mencionado, referente a la sección sobre Desarrollo e Implantación de una Política para el Profesorado:

Las cuestiones puestas de manifiesto en este informe van al fondo del trabajo del profesorado y de sus carreras profesionales. Para que cualquier reforma tenga éxito, es indispensable que los profesores sean ellos mismos protagonistas activos del desarrollo e implementación de las políticas educativas. Si los profesores no participan activamente en la formulación de esas políticas y no se apropian de ellas, es poco probable que se produzcan cambios sustanciales (OECD, 2005, p. 15).

Considerando los aspectos enunciados, tal vez haya llegado el momento de que las instituciones dedicadas a la formación del profesorado comprendan que a los docentes se 
les tiene que dar el poder para que puedan pasar a ser los principales autores de los discursos que son vitales en su profesión.

\section{Fabricating Europa}

Ya he tenido ocasión anteriormente de utilizar esta noción de fabricar Europa con el sentido atribuido por Nóvoa y Lawson (Mota, 2012). En el momento actual que se vive en este continente en el que todo parece estar en riesgo y en el que la educación artística es, sin duda, uno de los primeros campos en sufrir el ataque de los actuales gobiernos europeos, me parece todavía más importante evocar la noción de fabricating Europa (Nóvoa y Lawn, 2002) como un espacio educativo que puede incorporar de una nueva manera diferentes narrativas culturales, artefactos y símbolos nacionales. Si, por un lado, la cultura parece ser un concepto clave, los autores llaman nuestra atención sobre el peligro que existe de además incluir la negligencia, los silencios y las más variadas formas de exclusión.

El área de la «educación» puede ser encarada como un espacio para la construcción de nuevas identidades europeas, de organizaciones significativas transnacionales, de nuevas afinidades profesionales y de canales compartidos. Puede darnos la oportunidad para la legitimación de la desigualdad, de las soluciones neoliberales, también para oposiciones nacionalistas o basadas en el racismo que pretendan fortalecer las culturas nacionalistas. Europa puede ser un espacio de oposición a la globalización así como el hogar para los nuevos espacios cívicos. Pero también puede no serlo. (Nóvoa y Lawn, 2002).

La Educación Musical en la ES tiene algo que decir con respecto a la búsqueda de una cultura educativa europea que tenga en mente los peligros mencionados. En el contexto de la actual diversidad cultural en Europa, con una inmensa panoplia de existencias individuales desde el punto de vista cultural, social y económico, los programas de formación del profesorado de educación musical tienen que tener en su esencia el desarrollo de una sensibilidad intercultural, acompañada de la promoción de las competencias necesarias.

Si queremos contribuir al surgimiento y la construcción (fabrication) de Europa como un espacio en el que el diálogo intercultural vaya de la mano de políticas de inclusión social y cultural, podemos comenzar ya a dialogar sobre lo que eso significa para nuestra profesión en tanto que educadores musicales y para la educación musical propiamente dicha.

\section{Breve conclusión}

En este capítulo hemos intentado desarrollar algunos aspectos relacionados con la formación del profesorado de educación musical, contextualizados en la actual coyuntura europea y bajo una perspectiva crítica e informada por varios autores que se van asomando sistemáticamente a estas temáticas. He presentado también lo que considero el núcleo de una formación docente basada en principios democráticos y vinculada a una propuesta curricular que estaría permanentemente atenta al mundo de los niños y los jóvenes y a su natural pasión por la música.

No obstante, estamos ante un negro panorama en lo que respecta al sistema educativo, tanto en el nivel obligatorio de enseñanzas como en el superior. Los últimos datos presentados en Portugal sobre comunicación social, provenientes del Ministerio de Educación, divulgan datos terroríficos respecto al descenso de número de alumnos de
EB y ES: 13.000 alumnos menos en apenas un año en la EB ${ }^{3}$ y pérdida de casi 5.000 candidatos a la $\mathrm{ES}^{4}$. Por otro lado, el Ministerio llevó a cabo durante este año una reducción drástica del número de docentes en las escuelas propiciando el aumento del número de estudiantes por aula. Las consecuencias de estas políticas apuntan hacia lo que viene siendo una «educación de bajo coste» que determina una degradación del papel de la escuela pública y una preocupación por prestar únicamente un servicio más barato.

La ES está, de este modo, severamente amenazada y lo que podríamos considerar como una aproximación positiva de la renovación curricular en el contexto de Bolonia -y particularmente de la formación de profesores de Educación Musical-, puede estar corriendo el riesgo de no tener ningún significado.

Es en momentos como este en los que la resistencia es más necesaria y en los que la solidaridad entre las instituciones nacionales e internacionales asume una mayor relevancia. Por eso me gustaría terminar con una nota positiva que nos remite a la acción. Para ello me sirvo de una frase del músico de jazz y compositor António Pinho Vargas:

El momento del sufrimiento debe dar paso al momento de

la rebelión y al de la solidaridad activa. Es necesario actuar ahora $^{5}$.

\section{Notas}

${ }^{1}$ Para más información sobre este tema consultar http:// www.meloteca.com/escolas-profissionais-de-musica.htm. 2 Para más información, véase https://

estsass.wordpress.com/2011/04/20/moodlemoot-two-casesstudies-of-using-moodle-for-music-education/.

${ }^{3}$ http://www.publico.pt/sociedade/noticia/primeiro-cicloperde-40-mil-estudantes-em-sete-anos-1629260.

${ }^{4}$ http://expresso.sapo.pt/sociedade/numero-de-alunos-noensino-superior-nao-aumenta $=$ f893037.

${ }^{5}$ http://www.publico.pt/portugal/noticia/o-sofrimentodeve-dar-lugar-a-rebeliao-e-a-solidariedade-1579613.

\section{Referencias Citadas}

Abbs, P. (2003). Against the flow: Education, the arts and postmodern culture. Londres: Routledge.

Bergh, A. y Sloboda, J. A. (2010). Music and arts in conflict transformation: A review. Music $\&$ Arts in Action, 2(2), 2-17. Disponible en http://musicandartsinaction.net/ index.php/maia/article/view/conflicttransformation

Boal-Palheiros, G. y Encarnação, M. (2008). Music education as extra-curricular activity in Portuguese primary schools. En G. Mota y S. Malbrán (Ed.). Proceedings of the XXII ISME International Seminar on Research in Music Education. Oporto: ESE/FCT.

Bowman, W. (2007). Who is the "We"? Rethinking professionalism in music education. Action, Criticism, and theory for Music Education, 6(4), 109-131. Disponible en http://act.maydaygroup.org/articles/Bowman6_4.pdf

Dewey, J. (1916/2007). Democracy and education. Charleston: BiblioBazaar.

Dewey, J. (1934). Art as experience. Nueva York: Perigee Books.

Fernandes, D. (coord.) (2007). Estudo de avaliação do ensino artístico. Disponible en http://repositorio.ul.pt/handle/ $10451 / 5501$.

Giroux, H. A. (1989). Schooling for democracy: Critical pedagogy in the modern age. Londres: Routledge. 
Green, L. (2006). Popular music education in and for itself, and for 'other' music: current research in the classroom. International fournal of Music Education, 24(2), 101-118.

Greene, M. (1995). Releasing the imagination: Essays on education, the arts, and social change. San Francisco: Jossey-Bass.

Hutchings, P. y Huber, M. T. (2005). Building the Teaching Commons. Disponible en http:// www.carnegiefoundation.org/perspectives/buildingteaching-commons.

Jorgensen, E. (2003). Transforming music education. Indiana: Indiana University Press.

Jorgensen, E. (2008). The art of teaching music. Indiana: Indiana University Press.

Jorgensen, E. (2011). Pictures of music education. Indiana: Indiana University Press.

Madeira, T. (2002). O Ensino Articulado em Portugal. O olhar da Escola de Música de Leça da Palmeira. Trabajo Fin de Máster, inédito. Oporto: Escola Superior de Educação.

Mota, G. (2001). Portugal. En D. J. Hargreaves and A. C. North (Ed.). Musical development and learning: The international perspective. Londres: Continuum.

Mota, G. (2003a). A Educação Musical no mundo de hoje: um olhar crítico sobre a formação de professores. Revista do Centro de Educação da UFSM. Universidade Federal de Santa Maria, 28(2), 11-22.

Mota, G. $\left(2003^{b}\right)$. A formação para a expressão musical na educação de infância e no $1^{\circ}$ ciclo do ensino básico: contributo para um olhar crítico. Educare Apprendere, Lisboa, 1, 23-37.

Mota, G. (2003c). Pesquisa e formação em educação musical. Revista da ABEM, 8, 11-16.

Mota, G. (2007). A música no $1^{\circ}$ ciclo do ensino básico: Contributo para uma reflexão sobre o conceito de enriquecimento curricular. Revista de Educação Musical, 129, 16-21.

Mota, G. $\left(2008^{\mathrm{a}}\right)$. The involvement of young Portuguese people with Philharmonic Bands - a cultural and social perspective. En Proceedings of the 28th World Conference of the International Society for Music Education. Bolonia: ISME.

Mota, G. (Ed.) $\left(2008^{\mathrm{b}}\right)$. Crescer nas Bandas Filarmónicas um estudo sobre a construção da identidade musical de jovens portugueses. Oporto: Afrontamento.

Mota, G. (2012). Music in higher education after the Bologna Treaty: Or, in search of a new educational culture. En P. Schmidt y C. Benedict (Ed.). NSSE Yearbook, 111(1), 112132.

Nóvoa, A. y Lawn, M. (Ed.) (2002). Fabricating Europe. Londres: Kluwer Academic Publishers.

Nóvoa, A. (2007). O regresso dos Professores. Comunicación presentada en la Conference on Teacher Professional Development for the Quality and Equity of Lifelong Learning. Lisboa, del 27 al 29 de septiembre. Disponible en http:// hdl.handle.net/10451/687.

OECD (2005). Teachers matter: Attracting, developing and retaining effective teachers. OECD Publishing. Disponible en http:// w w w. o e c d. org / e d u c a ti o n / s c h o o l / attractingdevelopingandretainingeffectiveteachershomepage.htm.

Portugal (1983). Decreto-Lei $n^{\circ}$ 310/83. Disponible en http:/ / www.educacao-artistica.gov.pt/documentos / legisla $\%$ C3\% $\%$ A \%C3\%A3o/310-1983.pdf.
Portugal (1986). Lei de Bases do Sistema Educativo, Lei $\mathrm{n}^{\circ}$ 46/86, 1986. Disponible en http://www.dges.mctes.pt/ NR/rdonlyres / AE 6762 DF-1 DBF-40C0-B 194 E3FAA9516D79/1766/Lei46_86.pdf.

Portugal (1996). Dinâmicas, Memórias e Projectos das Escolas Profissionais. Oporto: Ministério da Educação, Departamento do Ensino Secundário.

Portugal (2002). Portaria $n^{\circ} 1550 / 2002$. Disponible en http: / / www.educacao-artistica.gov.pt/documentos / legisla $\% \mathrm{C} 3 \% \mathrm{~A} 7 \% \mathrm{C} 3 \% \mathrm{~A} 3 \mathrm{o} / 1550-2002$.pdf.

Portugal (2006). Decreto-Lei ${ }^{\circ}$ 74/2006 de 24 de Março, 2006. Disponible en http://alfa.fct.mctes.pt/apoios/ bolsas/DL_74_2006.pdf.

Portugal (2007). Decreto-Lei n ${ }^{\circ}$ 43/2007 de 22 de fevereiro, 2007. Disponible en http://www.dges.mctes.pt/DGES/ pt/Geral/MenuTopo/Legislacao/Formação+em+Áreas +Específicas.htm.

Portugal (2011). Revisão da estrutura curricular. Lisboa: Gabinete do Ministro da Educação e Ciência, 2011. Disponible en http://educar.files.wordpress.com/ 2011/12/revisao-curricular.pdf.

Wenger, E. (2006). Communities of practice. A brief introduction. Disponible en http://wenger-trayner.com/ introduction-to-communities-of-practice/. 


\section{Sobre la Autora}

\section{Graça Mota}

Graça Mota (pianista, máster en educación musical por la Universidad de Boston -EEUU-, y doctora por la Universidad de Keele, Reino Unido) es profesora coordinadora de la Unidad Técnico-Científica de Música en la Escuela Superior de Educación del Instituto Politécnico de Oporto (Portugal).

Es directora de la CIPEM (Centro de Investigación en Psicología de la Música y de la Educación Musical). Su trabajo de investigación se relaciona con la innovación en educación musical, desarrollo curricular y evaluación de la música, formación del profesorado, identidades musicales, narrativas musicales y práctica musical, e inclusión social. Una de las investigaciones actualmente en curso está financiada por la Funcación Calouste Gulbenkian y por la Fundación para la Ciencia y la Tecnología del Ministerio de Educación portugués.

Ha publicado en Portugal, EEUU, Reino Unido, Letonia, y Brasil. Recientemente ha escrito un capítulo titulado Narrative Soundings: An Anthology of Narrative Inquiry in Music Education dentro de un libro editado por Margaret Barret y Sandra Stauffer, y otro en coautoría con Sérgio Figueiredo (Brasil) en el Oxford Handbook of Music Education, editado por Gary McPherson y Graham Welch.

Fue Presidenta de la Comisión de Investigación de ISME durante el bienio 2008-2010.

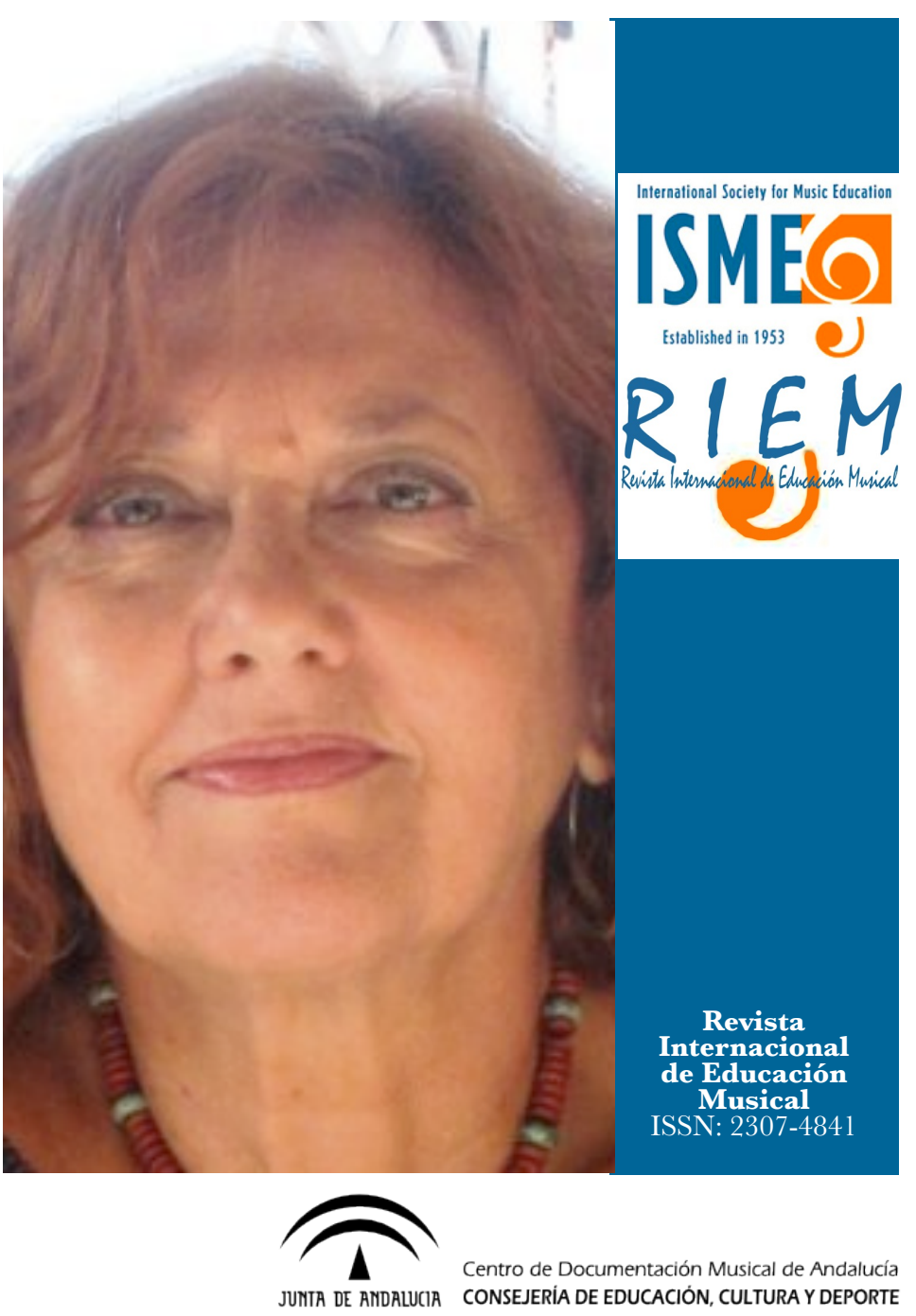

\section{Graça Mota}

Instituto Politécnico do Porto

Rua Dr. Roberto Frias, 602

4200-465 Porto. Portugal.

Gmota@ese.ipp.pt

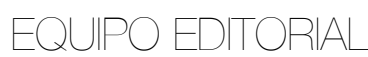

Editor:

José Luis Aróstegui Plaza, Universidad de Granada (España)

\section{Editora Adjunta:}

Rosa María Serrano Pastor, Universidad de Zaragoza (España)

\section{Consejo Editorial}

Carlos Abril, Universidad de Miami (Estados Unidos)

María del Carmen Aguilar, Instituto Coral de Buenos Aires (Argentina)

Miquel Alsina Tarrés, Universidad de Gerona (España)

Carmen Carrillo Aguilera. Universidad Internacional de Cataluña (España)

Sergio Luiz Figueiredo, Universidad del Estado de Santa Catarina (Brasil)

Patricia Adelaida González, Universidad Autónoma de Chihuahua (México)

Claudia Gluschankof, Instituto Levinsky (Israel)

Josep Gustems Carnicer, Universidad de Barcelona (España)

María Cecilia Jorquera Jaramillo, Universidad de Sevilla (España)

Gotzon Ibarretxe Txakartegi, Universidad del País Vasco (España)

Ana Laucirica Larrinaga, Universidad Pública de Navarra (España)

Ana Lucia Louro, Universidad Federal de Santa María (Brasil)
Isabel Cecilia Martínez, Universidad Nacional de La Plata (Argentina)

Teresa Mateiro, Universidad del Estado de Santa Catarina (Brasil)

María Teresa Moreno, Universidad Laval (Canadá)

Graça Boal Palheiros, Instituto de Educación de Oporto (Portugal)

Jèssica Pérez Moreno, Universidad Autónoma de Barcelona (España) y Universidad de Londres (Reino Unido)

Gabriel Enrique Rusinek Milner, Universidad Complutense de Madrid (España)

Patrick K. Schmidt, Universidad de Ontario Occidental (Canadá)

Favio Shifres, Universidad Nacional de La Plata (Argentina)

Maria dels Àngels Subirats Bayego, Universidad de Barcelona (España)

António Ângelo Ferreira Vasconcelos, Instituto de Educación de Setúbal (Portugal)

Maria Helena Vieira, Universidad del Miño (Portugal)

Gloria Patricia Zapata Restrepo, Universidad de Antioquía (Colombia) 